\title{
20. On analytical Transformations of a Reinhardt's Domain into itself.
}

\author{
By Masatsugu TsuJr.
}

(Received September 9, 1932.)

\section{Introduction.}

1. Let $x=x_{1}+i x_{2}, y=y_{1}+i y_{2}$, then Reinhardt's domain $D$ in a four dimensional space $\left(x_{1}, x_{2}, y_{1}, y_{2}\right)$ is defined as a domain, which is transformed. into itself by the transformations; $x^{\prime}=e^{i \alpha} x, y^{\prime}=e^{i \beta} y$, where $\alpha, \beta$ are any real numbers. The origin is the center of $D$. An analytical transformation of a domain $D$ into itself is a transformation of a form, $x^{\prime}=f(x, y), y^{\prime}=g(x, y)$ which with its inverse transformation, transforms $D$ into itself, where $f(x, y)$ and $g(x, y)$ are regular functions of $x$ and $y$ in $D$.

Reinhardt proved that if $x^{\prime}=f(x, y), y^{\prime}=g(x, y)$ is an analytical transformation of a bounded Reinhardt's domin $D$ into itself, the center being fixed, then $f(x, y)$ and $g(x, y)$ are homogeneous linear functions of $x$ and $y$ of special types.

In the following we denote (S) the aggregate of all analytical transformations of $D$ into itself so that the center is fixed.

Then Reinhardt's fundamental result is as follows:

Reindardt's theorem( ${ }^{1}$ ). (i) If $D$ is of the the form: $A|x|^{2}+B|y|^{2}<1$ $(A>0, B>0)$, then (S) consists of all transformations:

$$
\left.\begin{array}{l}
x^{\prime}=a x+\frac{b}{\lambda} y \\
y^{\prime}=c \lambda x+d y
\end{array}\right\}
$$

where $\lambda=\sqrt{\frac{A}{B}}$ and $|a|^{2}+|c|^{2}=1,|b|^{2}+|d|^{2}=1, a \bar{b}+c \bar{d}=0\left({ }^{2}\right)$.

(ii) if $D$ is a bounded Reinhardt's domain which is not of the above form, then (S) consists of all transformations:

( $\left.{ }^{1}\right)$ Reinhardt; Ueber Abbildungen durch analytische Funktionen zweier Veränder* lichen. (Math. Ann. 83 (1921) 211-255.)

( $\left.{ }^{2}\right)$ In the following we denote the conjugate complex of $z$ by $\bar{z}_{\text {. }}$ 


$$
\left.\begin{array}{l}
x^{\prime}=e^{i \alpha} x \\
y^{\prime}=e^{i \beta} y
\end{array}\right\}
$$

in general and in case when $D$ is symmetric, (S) contains outside (2) a transformation:

$$
\left.\begin{array}{l}
x^{\prime}=\mu e^{i \alpha} y \\
y^{\prime}=\frac{1}{\mu} e^{i \beta} x
\end{array}\right\}
$$

where $\mu$ is a certain constant.

Reinhardt's original proof is geometrical and complicated. $\mathrm{H}$. Cartan $\left({ }^{3}\right)$ gave another proof which is group-theoretical, and the proof is based on the theorems that any closed sub-group of a Lie's group is also a Lie's group (E. Cartan-v. Neumann's theorem) and that there exists an invariant positive definite Hermitian form for any closed Lie's group (Weyl's theorem).

In this paper we will give a quite elementary proof of Reinhardt's theorem without any use of such deep theorems.

That $f(x, y)$ and $g(x, y)$ are homogeneous linear functions of $x$ and $y$ can be proved very simply $\left({ }^{3}\right)$ so we will assume that $f(x, y)$ and $g(x, y)$ are linear. And H. Cartan $\left({ }^{3}\right)$ proved that (fs is a closed linear group, i, e, from a sequence $S_{1}, S_{2}, \ldots, S_{n}, \ldots$ of elements of $(S$, we can select a partial sequence which converges to an element of $\mathfrak{S}_{\text {. }}$

Hence we will assume in the following proof that

(S) is a closed linear group.

Evidently of contains transformations $S_{\alpha_{3}} ; x^{\prime}=e^{i \alpha} x, y^{\prime}=e^{i \beta} y$.

\section{Some Lemmas.}

2. Suppose $\mathfrak{G}$ contains a transformation;

$$
\left.S: \begin{array}{l}
x^{\prime}=a x+b y \\
y^{\prime}=c x+d y
\end{array}\right\}
$$

which is different from $S_{\alpha \beta}$.

We transform $S$ into a canonical form;

by a suitable non-singular matrix $T$.

$$
U=T S T^{-1}=\left(\begin{array}{ll}
\lambda_{1}, & b_{1} \\
0, & \lambda_{2}
\end{array}\right)
$$

( $\left.{ }^{3}\right)$ H. Cartan: Les functions de deux variables complexes et le problème de la representation analytique. (Journ. de math. (1931), 1-114.) 
Then

$$
U=T S T^{-1}=\left(\begin{array}{cc}
\lambda_{1}, & b_{1} \\
0, & \lambda_{2}
\end{array}\right) \quad(n=0, \pm 1, \pm 2, \ldots) .
$$

Since SS $^{\prime}=T\left(S T^{-1}\right.$ is a closed group, we conclude that $\left|\lambda_{1}\right|=1,\left|\lambda_{2}\right|=1$ by making $n \rightarrow \infty$ or $n \rightarrow-\infty$ in (5). Since $\lambda_{1}, \lambda_{2}$ are the roots of the characteristic equation of $S$ :

we have

$$
\lambda^{2}-(a+d) \lambda+a d-b c=0
$$

$$
\begin{gathered}
|a d-b c|=\left|\lambda_{1} \lambda_{2}\right|=1 \\
|a+d|=\left|\lambda_{1}+\lambda_{2}\right| \leqq\left|\lambda_{1}\right|+\left|\lambda_{2}\right|=2
\end{gathered}
$$

Since $S_{z}=\left(\begin{array}{ll}a z, & b \\ c z, & d\end{array}\right)(|z|=1)$ belongs to $\mathcal{G}$, we get from $(7),|a z+d| \leqq 2$. Choose $z$, such that $\arg a z=\arg d$, then we get $|a|+|d| \leqq 2$.

Hence we have

Lemma 1. If $S=\left(\begin{array}{ll}a, & b \\ c, & d\end{array}\right)$ belongs to $\mathbb{S}$ and $\lambda_{1}, \lambda_{2}$ are the roots of the characteristic equation of $S$, then

$$
\begin{aligned}
& \left|\lambda_{1}\right|=1, \quad\left|\lambda_{2}\right|=1 \\
& |a d-b c|=1 \\
& |a|+|d| \leqq 2 .
\end{aligned}
$$

3. Since the determinant of $S_{z}$ is $z$ times the determinant of $S$, by a suitable choice of $z(|z|=1)$, we may assume in $S$,

$$
\Delta=a d-b c=1 \text {. }
$$

Now the characteristic equation of $S_{z}$ is

$$
\lambda^{2}-(\alpha z+d) \lambda+z=0
$$

or rewriting

$$
w=\frac{z}{\lambda}=\frac{\lambda-d}{a \lambda-1}
$$

Consider $w$ as a function of $\lambda$, then by Lemma 1 , w lies on $|w|=1$ when $\lambda$ moves on $|\lambda|=1$.

In (10), two cases occur according as $w$ is a constant or not.

(i) $\quad w=\frac{z}{\lambda}=$ const $=w_{0} \quad\left(\left|w_{0}\right|=1\right)$.

Then $\frac{\lambda-d}{a \lambda-1}=w_{0}$ for every $\lambda$ on $|\lambda|=1$. Hence $w_{0} d=1, d=w_{0}$. 
or $d=\bar{a},|a|=1, a d=1$.

From $a d-b c=1$, we get $b c=0$. Hence $b=0$ or $c=0$.

If $c=0$, then $S=\left(\begin{array}{ll}a, & b \\ 0, & a\end{array}\right)$.

Since $S_{1}=\left(\begin{array}{c}a, b z \\ 0, \bar{a} z\end{array}\right)(|z|=1)$ belongs to $(\mathfrak{S}$, we choose $z$ so that $\bar{a} z=\alpha$, then $S_{1}=\left(\begin{array}{ll}a, & b_{1} \\ 0, & a\end{array}\right)\left(b_{1}=b z\right)$ belongs to $\mathfrak{S S}$. Now $S_{1}{ }^{n}=\left(\begin{array}{rr}a^{n}, & n a^{i n-1} b_{1} \\ 0, & a^{n}\end{array}\right)$, hence if $b \neq 0,\left|n a^{n-1} b_{1}\right| \rightarrow \infty$ for $n \rightarrow \infty$, which contradicts that (s) is closed, so that $b=0$.

Hence if $w=$ const., $S$ must be of the form: $S=\left(\begin{array}{ll}a, & 0 \\ 0, & \bar{a}\end{array}\right)(|a|=1)$. We reach to the same result if we assume $b=0$, instead of $c=0$.

(ii) $w \neq$ const.

In this case, $w=\frac{\lambda-d}{a \lambda-1}$ moves on the unit circle, when $\lambda$ moves on $|x|=1$, so that

$$
|a| \neq 1, \quad d=\bar{a}
$$

By Lemma 1,

$$
2|a|=|a|+|d| \leqq 2 \text { or }|a| \leqq 1 \text {. }
$$

Since the equaltity is excluded,

$$
|a|<1, \quad d=\bar{a}_{0}
$$

Lemma 2. If $S=\left(\begin{array}{ll}a, & b \\ c, & d\end{array}\right)(a d-b c=1)$ belongs to $\mathbb{S}$, then either

$$
\text { (i) } \quad S=\left(\begin{array}{ll}
a, & 0 \\
0, & \frac{a}{a}
\end{array}\right) \quad(|a|=1)
$$

or

(ii) $\quad S=\left(\begin{array}{ll}a, & b \\ c, & \bar{a}\end{array}\right) \quad(|a|<1, \quad b c \neq 0)$.

4. Next we will investigate the case (ii) and suppose that $a b c \neq 0$. We make the change of variables;

$$
\left.T: \quad \begin{array}{l}
X=\lambda x \\
Y=y
\end{array}\right\}
$$

By $T, D$ is transformed into $D^{\prime}$, which is evidently a bounded Reinhardt's domain, and is transformed into itself by a group $\mathbb{G S}^{\prime}=T\left(\mathbb{S}^{5} T^{-1}\right.$. 
By (11), $S=\left(\begin{array}{l}a, b \\ c, \frac{a}{a}\end{array}\right)$ becomes

$$
\left.S_{1}: \quad \begin{array}{l}
X^{\prime}=a X+\lambda b Y \\
Y^{\prime}=\frac{c X}{\lambda}+\bar{a} Y
\end{array}\right\} \quad(a \bar{a}-b c=1, \quad|a|<1, \quad b c \neq 0)
$$

We will show that a real $\lambda$ can be chosen so that

or

$$
\left.\left|X^{2}+\right| Y^{\prime}\right|^{2}=|X|^{2}+|Y|^{2}
$$

$$
a \bar{a}+\frac{c \bar{c}}{\lambda^{2}}=1, \quad a \bar{a}+\lambda^{2} b \bar{b}=1, \quad \bar{b} \lambda+\frac{c}{\lambda}=0 .
$$

In fact, we get $\lambda^{2}=\frac{|c|^{2}}{1-|a|^{2}}(>0)$ from the first equation of (13), and this $\lambda$ satisfy the remaining equations of (13).

Hence for such a $\lambda, S_{1}$ becomes a unitary matrix with a determinant 1. If we make correspond to $S=\left(\begin{array}{l}a, b \\ c, d\end{array}\right)$ of $\mathcal{B S}^{\circ}$, a transformation $\sigma: z^{\prime}=\frac{a+b z}{c+d z}$ on the $z$-plane or on the Riemann's sphere $K$, then we get a group $g^{\prime}$ of transformations of $K$. If $S$ is a unitary transformation with determinant unity, then $\sigma$ is a rotation of $K$ about its diameter. Since $\mathfrak{G S}^{\prime}$ contains $S_{\alpha \beta}, \mathfrak{g}^{\prime}$ contains $S_{\theta}: z^{\prime}=z e^{i \theta}(0 \leqq \theta \leqq 2 \pi)$ or all rotations about the diameter through the north pole. And $g^{\prime}$ contains another

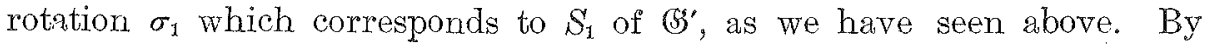
a geometrical consideration, we can easily see that all rotations of $K$ can be obtained by a suitable combination of $S_{\theta}$ and $\sigma_{1}$. Hence (G) contains all unitary transformations with determinant unity.

Lemma 3. If $S=\left(\begin{array}{ll}a, & b \\ c, & \bar{a}\end{array}\right)(a \bar{a}-b c=1, a b c \neq 0)$ belongs to $\mathcal{S}$, we can find a real $\lambda$, such that $\mathscr{G}^{\circ}=\left(\begin{array}{ll}\lambda, & 0 \\ 0, & 1\end{array}\right) \mathscr{S}\left(\begin{array}{ll}\lambda, & 0 \\ 0, & 1\end{array}\right)^{-1}$ contains all unitary transformations with determinant unity.

5. We prove the following lemmas only for completeness.

Lemma 4. If $\left|X^{\prime}\right|^{2}+\left|Y^{\prime}\right|^{2}=|X|^{2}+\mid Y^{2}$, there exists a unitary transformation with determinant unity, which transforms $(X, Y)$ to $\left(X^{\prime}, Y^{\prime}\right)$.

Proof. Let

$$
\left.\begin{array}{l}
X^{\prime}=a X+b Y \\
Y^{\prime}=-\bar{b} X+\bar{a} Y
\end{array}\right\}\left(|a|^{2}+|b|^{2}=1\right)
$$


be such a transformation, then

$$
\bar{Y}^{\prime}=a \bar{Y}-b \bar{X}
$$

Solving for $a, b$, we get

$$
a=\frac{\bar{X} X^{\prime}+Y \bar{Y}^{\prime}}{\left.\left|X^{2}+\right| Y\right|^{2}}, \quad b=\frac{\bar{Y} X^{\prime}-X \bar{Y}^{\prime}}{|X|^{2}+\mid Y^{2}}
$$

and these $a, b$ satisfy the condition $|a|^{2}+|b|^{2}=1$.

Lemma 5. If

$$
\left.\begin{array}{l}
X^{\prime}=a X+b Y \\
Y^{\prime}=c X+d Y
\end{array}\right\}
$$

transforms $|X|^{2}+\mid Y^{2}<R$ into itself, then

$$
|a|^{2}+|c|^{2}=1, \quad|b|^{2}+|d|^{2}=1, \quad a \bar{b}+c \bar{d}=0 .
$$

Proof. Since (14) transforms the boundary $|X|^{2}+|Y|^{2}=R$ into itself,

$$
\left|X^{\prime}\right|^{2}+\left|Y^{o}\right|^{2}=R \quad \text { if } \quad|X|^{2}+|Y|^{2}=R \text {. }
$$

Now

$$
\begin{aligned}
\left|X^{\prime \prime}\right|^{2}+\left|Y^{\prime}\right|^{2}=\mid & \left.X\right|^{2}(a \bar{a}+c \bar{c})+|Y|^{2}(b \bar{b}+d \bar{d}) \\
& +X \bar{Y}(a \bar{b}+c \bar{d})+\bar{X} Y(\bar{a} \bar{b}+\bar{c} d) .
\end{aligned}
$$

Put $(X, Y)=(\sqrt{R}, 0)$ and $(0, \sqrt{R})$ in (16), we get by (15),

$$
a \bar{a}+c \bar{c}=1, \quad b \bar{b}+d \bar{d}=1,
$$

so that on $|X|^{2}+|Y|^{2}=R$,

$$
X \bar{Y}(a \bar{b}+c \bar{d})+\bar{X} Y(\bar{a} b+\bar{c} d)=0 .
$$

Putting $(X, Y)=\left(\sqrt{\frac{R}{2}}, \sqrt{\frac{R}{2}}\right)$ and $\left(\sqrt{\frac{R}{2}}, i \sqrt{\frac{R}{2}}\right)$ in (16), we have

$$
a \bar{b}+c \bar{d}=0, \quad \text { q.e.d. }
$$

\section{Proof of Reinhardt's Theorem.}

6. Suppose $\&$ contains $S=\left(\begin{array}{ll}a, & b \\ c, & \bar{a}\end{array}\right) \quad(a \bar{a}-b c=1, a b c \neq 0)$, then by Lemma 3, $\mathfrak{S}^{*}=\left(\begin{array}{ll}\lambda, & 0 \\ 0, & 1\end{array}\right)$ GS $\left(\begin{array}{ll}\lambda, & 0 \\ 0, & 1\end{array}\right)^{-1}$ contains all unitary transformations with determinant unity. Let $D$ be transformed into $D^{\prime}$ by $X=\lambda x, Y=y$, then we will show that $D^{\prime}$ coincides with $|X|^{2}+|Y|^{2}<R$.

To prove this, let the upper limit of $|X|^{2}+|Y|^{2}$ in $D^{\prime}$ be $R$, then there exists a point $\left(X_{0}, Y_{0}\right)$ in $D^{\prime}$, such that $\left|X_{0}\right|^{2}+\left|Y_{0}\right|^{2}=R-\varepsilon(\varepsilon>0)$ and a continuous curve $L ; X=X(t), Y=Y(t)(0 \leqq t \leqq 1)$ which connects the origin to $\left(X_{0}, Y_{0}\right)$ in $D^{\prime}$. 
Through every point $(X(t), Y(t))$ on $L$, there passes a sphere;

$$
|X|^{2}+|Y|^{2}=|X(t)|^{2}+|Y(t)|^{2}
$$

By Lemma 4, there exists a unitary transformation with determinant unity which transforms $(X(t), Y(t))$ to any $(X, Y)$ on this sphere and since such a transformation belongs to $\mathcal{F}^{\prime \prime}$, we see that the whole sphere (17) and hence $|X|^{2}+|Y|^{2} \leqq R-\varepsilon$ belongs to $D^{\prime}$. Since $\varepsilon$ is arbitary, $|X|^{2}+|Y|^{2}<R$ belongs to $D^{\prime}$. But $R$ is the upper limit of $|X|^{2}+|Y|^{2}$ in $D^{\prime}$, so that $D^{\prime}$ coincides with $|X|^{2}+\mid Y^{2}<R$.

By Lemma 5, all linear transformations which transforms $|X|^{2}+|Y|^{2}$ $<P$ into itself are known, i. e.,

$$
\left.\begin{array}{l}
X^{\prime \prime}=a X+b Y \\
Y^{\prime}=c X+d Y
\end{array}\right\}
$$

where $|a|^{2}+|c|^{2}=1,|b|^{2}+|c|^{2}=1, a \bar{b}+c \bar{d}=0$.

Returning to $D, D$ is of the form; $\lambda^{2}|x|^{2}+|y|^{2}<R$ and is transformed into itself by the transformations,

$$
\left.\begin{array}{c}
x^{\prime}=a x+\frac{b}{\lambda} y \\
y^{\prime}=c \lambda x+d y
\end{array}\right\}
$$

Hence the first part of the theorem is proved.

To prove the second part, suppose (s) contains transformations with determinant unity only of the form; $S=\left(\begin{array}{cc}a, & b \\ c, & \bar{a}\end{array}\right) \quad(a \bar{a}-b c=1, a b c=0)$, then by Lemma 2, such a $S$ must be of the form; $\left(\begin{array}{cc}a, & 0 \\ 0, & a\end{array}\right)\left(|a|^{2}=1\right)$ or $\left(\begin{array}{ll}0, & b \\ c, & 0\end{array}\right)(b c=-1)$

If we take off the limitation that the determinant is unity, (S) contains only transformations of the form; $\left(\begin{array}{cc}e^{i \alpha}, & 0 \\ 0, & e^{i \beta}\end{array}\right)$ or $\left(\begin{array}{cc}0, & \mu e^{i \alpha} \\ \frac{1}{\mu} e^{i \beta}, & 0\end{array}\right)$.

And in the latter transformation, $\mu$ can take only one certain value. For if two transformations $\left(\begin{array}{c}0, \mu_{1} e^{i \alpha_{1}} \\ \frac{1}{\mu_{1}} e^{i \beta_{1}}, 0\end{array}\right),\left(\begin{array}{c}0, \mu_{2} e^{i \alpha_{2}} \\ \frac{1}{\mu_{2}} e^{i \beta_{2}}, 0\end{array}\right)$ exist, then 
their product $\left(\begin{array}{cc}\frac{\mu_{1}}{\mu_{2}} e^{i\left(\alpha_{1}+\beta_{2}\right)}, & 0 \\ 0, & \frac{\mu_{2}}{\mu_{1}} e^{i\left(\alpha_{2}+\beta_{1}\right)}\end{array}\right)$ belongs to SS, so that by Lemma 1 $\left|\frac{\mu_{1}}{\mu_{2}}\right|=1$ or $\left|\mu_{1}\right|=\left|\mu_{2}\right|$.

Hence the second part is proved.

I will express my cordial thanks to Prof. Kakeya for the valuable advices and suggestions.

Mathematical Institute, Imperial University of Tokyo. 